\title{
Development of Work Breakdown Structure (WBS) Standard for Producing the Risk Based Structural Work Safety Plan Of Building
}

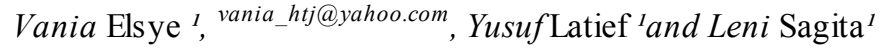 \\ 1 Civil Engineering Department, Engineering Faculty, University of Indonesia, Depok, West Java, 16424, Indonesia
}

\begin{abstract}
A construction project is inseparable from a series of tasks in the form of activities. Activities that take place on a project can be disrupted due to various reasons, one of the causes of the dis ruption of project activities are accidents on construction projects. The risk of workplace accidents can be prevented with early identification and analys is of the potential danger that exist in every activity contained in the project's WBS. The need for a standardized WBS in preventing the risk of workplace accidents is very important because itwould present a risk assessment, impact and frequency arising from workplace accidents. The aim of this study is to develop a risk-based WBS standard particularly for structural work, by using a qualitative approach. The results of this research are WBS standard for building, source of potentially dangerous risk at structural work,and the development of safety plan using a risk-based WBS that has been standardized, as a form of prevention, reduction or even nullify the risk of workplace accidents (to obtain zero accidents) during construction project implementation.
\end{abstract}

\section{Introduction}

Construction industry is a very unique industry and more dangerous than other industries. The construction sites are constantly changing and temporary. Each construction sites involve of many sub contractors and they perform different types of work in close proximity to each other. The safety of workplace is an essential component of efficiency and productivity[6]. Construction workers, both staff and field workers are protected assets in order to work properly and productively until construction is completed without any workplace accident (zero accident). Workers need to be protected against the threat of accidents and health in work. Occupational safety measures are indispensable, both to workers and to corporations and to their production. Workers must follow safety-related regulations, in this case the OSH program, as the OSH program is closely related to the safety of the workers. A comprehensive OSH program and self-awareness of workers is essential to reduce the number of accidents [2].

Work accidents occurring on a project can actually be minimized, if activities on the project are well defined and each job can be placed at a level and level corresponding to a standard WBS, WBS constructing used during the project phase with a basic scope that has been identified. Initially, WBS was created with a restricted formation of scope, however WBS will be reviewed in addition to scope information developed or developed through complete project-to-project analysis. To complete the description of WBS and its application refers based on standard practical guidance according to the principle used so that will get high quality WBS [8], that is easy to determine the amount of activity in project.

Each defined WBS level brings WBS to a more complex level of activity, each activity that has been grouped in a standard WBS, will be easier to identify and mitigate its potential risks, so it is important to develop a standard Work Breakdown Structure (WBS) for the manufacture of safety based risk [11] to obtain output that can be used as a guideline for the implementation of construction.

\section{Research Objectives}

The objectives of this research are :

1. To develop of building standardized work breakdown structure.

2. To identify a potentially dangerous source of

risk for structure work.

3. To develop safety plan using a risk-based WBS that has been standardized.

\section{$3 \quad$ Literature Review}

\subsection{Work Breakdown Structure}

Work Breakdown Structure is a resultsoriented analysis of the work covered in a project called the total scope of the project. WBS is a fundamental document in project management as it provides the basis for planning and managing the schedules, costs and changes that occur. WBS serves as a deliverable oriented tool [4]. WBS formulation enables the project to be better in its definition, both in terms of resources and the estimated time required to complete the project to be further enhanced. However, it is important to note that WBS projects change over time, depending on the needs and constraints that the project is experiencing [10].

WBS forms the basis for planning, estimating, scheduling, monitoring, management and control of all project activities. The proposed methods for developing a deliverable are well defined and comprehensive, and the importance of increasing the 
probability of project success by ensuring that the best resources applied to the project [12]. A standardized WBS for the project is used to facilitate the preparation of project financing and a standardized WBS is also used to define project activities [1].

The best method of constructing WBS is the hierarchically displayed decomposition of the entire scope that the project team will undertake to complete the project objectives and produce the intended work output. In PMBOK 5th Edition, 2013 The planned work at the bottom of the WBS called work package. (deliverables) and not the activities.

\subsection{Risk Management}

Risk management is an important part of the decision-making process in construction project management, especially regarding integration, scope, time, cost, quality, human resources, communications and project procurement. Creating hierarchical risk management with RBS can improve future project prospects for identifying uncertainty and probabilities [13]. Risk is considered a negative term, but in the engineering construction industry in managing risks that arise is necessary and implemented in a structured way, knowledge of risk management, can nullify and minimize risks occurring in construction projects [7].

\section{3 \\ Performance Occupational Safety and Health}

of

Accidents occurring at construction sites may result in serious physical injuries and accidents such as people falling from altitudes and crashing material or equipment, the cause of occupational accidents may occur due to worker negligence and also undisciplined workers in the project environment, preventive measures against accidents should Implemented and implemented, so that workers and top management will raise awareness in preventing The workplace hazard handling strategy for construction may introduce potential hazards [5], further investigation of frequent hazards and may result in safety plans in construction projects

Safety Plan is a plan document containing practical safety that can assist companies in avoiding potential hazards and can control them in the best way when in these hazardous conditions, the importance of safety planning training during the planning, design and implementation phase of a project because it is able to minimize the risk of construction projects and Can improve workers' safety.

\section{$4 \quad$ Methodology}

This research is using qualitative approach to achieve formulation of standardised WBS for building construction. A survey and deep interview was also conducted by means of a structured questionnaire to contractor's experts who have had more than 20 years experiences in highrise building construction projects. Here in Figure 1. The Research Flow, there is a sequence of implementation of this study.

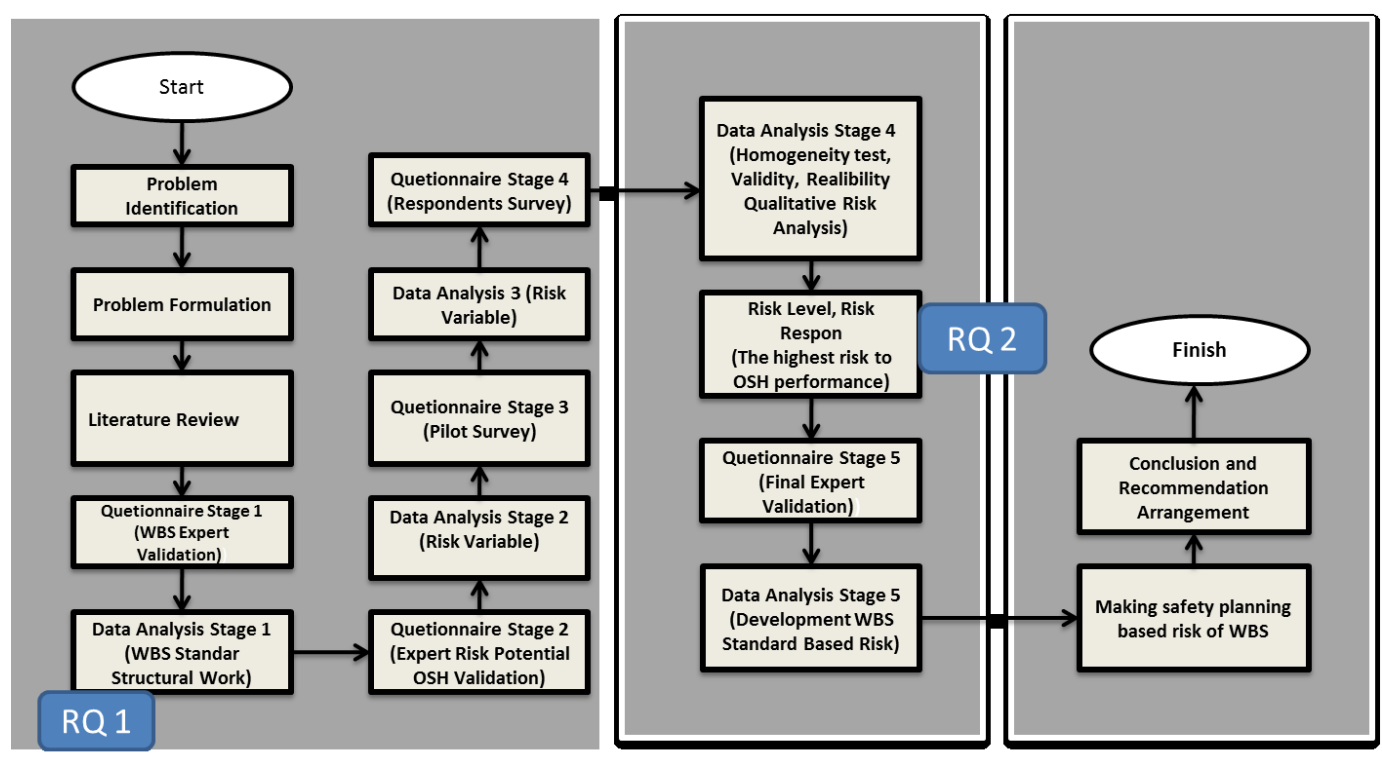

Fig. 1. The Research Flow

\subsection{Questionnaire}

In this study there are 4 (four) questionnaires to be made: questionnaires in stage I, II, questionnaires in stage III, questionnaire at stage IV, and questionnaire at stage $\mathrm{V}$.
- Questionnaire in phase I is with expert form validation related to standard WBS level will be done variable validation by expert first. There is also the number of experts / experts are 3-5 people with a minimum of 10 years 
experiences in the field of high-rise construction and education $\mathrm{S} 1$. The validation process contains expert responses to agree / disagree that variable $\mathrm{X}$ is an activity in highrise building.

- Questionnaires in phase II will be validated variables by experts in advance related risk factors that affect OSH. There is also the number of experts / experts are 3-5 people with a minimum of 10 years experiences in the field of high-rise construction and education S1.Proses validation contains expert responses upon agree / disagree that the variable $\mathrm{X}$ effect on the risk of high building construction $\mathrm{OSH}$

- After obtained a valid X variable by expert / expert, conducted phase III that is pilot survey to know whether questionnaire made easy understood responder. Pilot survey is in the form of questionnaires to be distributed to 5 10 respondents. Here is an example of a questionnaire in stage III.

- Questionnaires in stage IV are used to obtain the highest risk factors that affect the quality performance of high construction work. This questionnaire will be distributed to respondents who are / have worked in the process of building high-rise project and handling high building construction work with experience of at least 5 years. Here is an example of a stage IV questionnaire.

- Questionnaire at stage V is expert validation aimed at soliciting expert responses related to the results of risk analysis while discussing the causes, preventive actions, impacts, and corrective actions of the highest risk on the process of implementing high-rise building construction work. Here is an example of a stage $\mathrm{V}$ questionnaire.

\subsection{Respondent}

A total of 35 respondents, the profile of respondent is defined in Table 1. Profile of Respondents as follows:

Table 1. Profile of Respondents

\begin{tabular}{|c|l|c|}
\hline No & \multicolumn{1}{|c|}{ Description } & Total \\
\hline 1 & Position & \\
& Staff/Engineering & 28 \\
& Manager & 6 \\
& $>$ Manager & 1 \\
\hline 2 & Work Experience & \\
& $<5$ years & 19 \\
& 5 - 10 years & 7 \\
& $>10$ years & 9 \\
\hline 3 & Education & \\
\hline
\end{tabular}

\begin{tabular}{|l|l|c|}
\hline No & Description & Total \\
\hline & D3 & 2 \\
& S1 & 31 \\
& S2 & 2 \\
\hline
\end{tabular}

In homogenity test with Krusskal Wallis test, according to position, work experience, and education, the result is ho $>0,05$.

WBS research variables the standard derived from the archive analysis of the WBS work package from 5 high construction project data from the structural work down to the top structure in the last 5 years there are 31 work packages, and based on expert validation of the potential risks that affect the performance of OSH 173 risk variables. Then the variable is defined in Table 2 . Research Variables as follows:

Table 2. Research Variables

\begin{tabular}{|c|c|c|c|}
\hline \multicolumn{2}{|c|}{$\begin{array}{l}\text { WBS LEVEL } 6 \\
\text { Activities }\end{array}$} & $\begin{array}{l}\text { RIS K VARIAB } \\
\text { INFLUENCING }\end{array}$ & $\begin{array}{ll}\text { ES } \\
\text { OSH }\end{array}$ \\
\hline \multicolumn{4}{|c|}{$\begin{array}{c}\text { Work Package: Land Clearing Method: Conventional } \\
\text { (Land Work) }\end{array}$} \\
\hline \multirow{3}{*}{ Clearing } & $\mathrm{X} 1$ & $\begin{array}{l}\text { Workers attacked } \\
\text { wild animals in the } \\
\text { area around land } \\
\text { clearing }\end{array}$ & \\
\hline & $\mathrm{X} 2$ & $\begin{array}{l}\text { Feet affected by } \\
\text { hoe at the time of } \\
\text { land clearing }\end{array}$ & \\
\hline & $\mathrm{X} 3$ & $\begin{array}{l}\text { Hands exposed to } \\
\text { sickle }\end{array}$ & \\
\hline $\begin{array}{c}\text { Land } \\
\text { Alignment }\end{array}$ & $\mathrm{X} 4$ & $\begin{array}{l}\text { Feet affected by } \\
\text { hoe at the time of } \\
\text { land clearing }\end{array}$ & \\
\hline \multicolumn{4}{|c|}{ Work Package: Land Clearing Method: Mechanical } \\
\hline \multirow{3}{*}{ Clearing } & $\mathrm{X} 5$ & $\begin{array}{l}\text { Workers sprinkled } \\
\text { or doused with } \\
\text { herbicide solution }\end{array}$ & \\
\hline & $\mathrm{X} 6$ & $\begin{array}{l}\text { Tools crashing the } \\
\text { workers / facilities } \\
\text { around (backhoe) } \\
\text { at the time of land } \\
\text { clearing }\end{array}$ & \\
\hline & X7 & $\begin{array}{l}\text { Slip due to the } \\
\text { condition of the } \\
\text { steep soil }\end{array}$ & \\
\hline \multirow{2}{*}{$\begin{array}{c}\text { Land } \\
\text { Alignment }\end{array}$} & $\mathrm{X} 8$ & $\begin{array}{l}\text { Accidents during } \\
\text { machine } \\
\text { mobilization }\end{array}$ & \\
\hline & X9 & $\begin{array}{l}\text { Tool crashing into } \\
\text { nearby worker / } \\
\text { facility (bulldozer }\end{array}$ & \\
\hline
\end{tabular}




\begin{tabular}{|c|c|c|c|}
\hline \multicolumn{2}{|c|}{$\begin{array}{l}\text { WBS LEVEL } 6 \\
\text { Activities }\end{array}$} & \multicolumn{2}{|c|}{$\begin{array}{l}\text { RISK VARIABLES } \\
\text { INFLUENCING OSH }\end{array}$} \\
\hline & & $\begin{array}{l}\text { and combine } \\
\text { harvester) at } \\
\text { ground level }\end{array}$ & \\
\hline \multicolumn{4}{|c|}{ Work Package: Dewatering } \\
\hline \multirow{4}{*}{$\begin{array}{l}\text { Channel } \\
\text { Preparation }\end{array}$} & $\mathrm{X} 10$ & $\begin{array}{l}\text { Tool hit the worker } \\
\text { / facility } \\
\text { around(excavator) }\end{array}$ & \\
\hline & $\mathrm{X} 11$ & $\begin{array}{l}\text { Struck by a stone } \\
\text { as a shade }\end{array}$ & \\
\hline & $\mathrm{X} 12$ & $\begin{array}{l}\text { Workers are } \\
\text { dropped due to } \\
\text { slippery area } \\
\text { conditions in the } \\
\text { channel } \\
\text { preparation }\end{array}$ & \\
\hline & $\mathrm{X} 13$ & $\begin{array}{l}\text { Injured by } \\
\text { equipment }\end{array}$ & \\
\hline Excavation & $\mathrm{X} 14$ & $\begin{array}{l}\text { Excavation } \\
\text { crashing the } \\
\text { worker / facility } \\
\text { around (backhoe) }\end{array}$ & \\
\hline \multirow{2}{*}{ Excavation } & $\mathrm{X} 15$ & $\begin{array}{l}\text { Worker fell into } \\
\text { the excavation }\end{array}$ & \\
\hline & $\mathrm{X} 16$ & $\begin{array}{l}\text { Landslide } \\
\text { excavation }\end{array}$ & \\
\hline \multirow{2}{*}{$\begin{array}{l}\text { Installation of } \\
\text { submeasible } \\
\text { pumps }\end{array}$} & $\mathrm{X} 17$ & $\begin{array}{l}\text { Workers are } \\
\text { electrocuted from } \\
\text { the pumping } \\
\text { machine wires }\end{array}$ & \\
\hline & $\mathrm{X} 18$ & $\begin{array}{l}\text { Workers fall / slips } \\
\text { due to slippery } \\
\text { conditions on } \\
\text { pump installation }\end{array}$ & \\
\hline $\begin{array}{l}\text { Making } \\
\text { Well Point }\end{array}$ & $\mathrm{X} 19$ & $\begin{array}{l}\text { Workers exposed } \\
\text { to water runoff } \\
\text { during the making } \\
\text { of well points }\end{array}$ & \\
\hline $\begin{array}{l}\text { Making } \\
\text { Well Test }\end{array}$ & $\mathrm{X} 20$ & $\begin{array}{l}\text { Workers fall due to } \\
\text { slippery areas } \\
\text { around the well }\end{array}$ & \\
\hline \multirow{6}{*}{$\begin{array}{l}\text { Channel } \\
\text { Preparation }\end{array}$} & $\mathrm{X} 21$ & $\begin{array}{l}\text { Transfer of } \\
\text { existing channel }\end{array}$ & \\
\hline & $\overline{\mathrm{X} 22}$ & Stricken casing & \\
\hline & $\mathrm{X} 23$ & $\begin{array}{l}\text { Tools hit the } \\
\text { workers / facilities } \\
\text { around (excavator) }\end{array}$ & \\
\hline & $\mathrm{X} 24$ & $\begin{array}{l}\text { Injured because of } \\
\text { equipment }\end{array}$ & \\
\hline & $\mathrm{X} 25$ & $\begin{array}{l}\text { Affected crane } \\
\text { maneuver }\end{array}$ & \\
\hline & $\mathrm{X} 26$ & $\begin{array}{l}\text { Hit the mobile } \\
\text { crane }\end{array}$ & \\
\hline
\end{tabular}

\begin{tabular}{|c|c|c|c|}
\hline \multicolumn{2}{|c|}{$\begin{array}{c}\text { WBS LEVEL } 6 \\
\text { Activities }\end{array}$} & \multicolumn{2}{|c|}{$\begin{array}{c}\text { RIS K VARIABLES } \\
\text { INFLUENCING OSH }\end{array}$} \\
\hline & $\mathrm{X} 27$ & $\begin{array}{l}\text { Material regardless } \\
\text { of the hook / sling } \\
\text { mobile crane is } \\
\text { broken }\end{array}$ & \\
\hline & $\mathrm{X} 28$ & $\begin{array}{l}\text { Tools crashing } \\
\text { workers / facilities } \\
\text { around }\end{array}$ & \\
\hline & $\mathrm{X} 29$ & $\begin{array}{l}\text { Workers fall into } \\
\text { the excavation }\end{array}$ & \\
\hline & $\mathrm{X} 30$ & $\begin{array}{l}\text { Landslide } \\
\text { excavation }\end{array}$ & \\
\hline \multicolumn{4}{|c|}{ Work Packages: Digging and Landing } \\
\hline \multirow{2}{*}{$\begin{array}{l}\text { Slope } \\
\text { Reinforcement }\end{array}$} & $\mathrm{X} 31$ & $\begin{array}{l}\text { Landslides/side } \\
\text { wall collapse }\end{array}$ & \\
\hline & $\mathrm{X} 32$ & $\begin{array}{l}\text { Workers fall due to } \\
\text { slope } \\
\text { reinforcement } \\
\text { activity }\end{array}$ & \\
\hline \multirow{3}{*}{ Excavation } & $\mathrm{X} 33$ & $\begin{array}{l}\text { Tools crashing } \\
\text { workers / facilities } \\
\text { around }\end{array}$ & \\
\hline & X34 & $\begin{array}{l}\text { Workers fall into } \\
\text { the excavation }\end{array}$ & \\
\hline & $\mathrm{X} 35$ & $\begin{array}{l}\text { Landslide } \\
\text { excavation }\end{array}$ & \\
\hline Landscapes & $\mathrm{X} 36$ & $\begin{array}{l}\text { Workers fell / } \\
\text { stumbled while } \\
\text { throwing away the } \\
\text { soil }\end{array}$ & \\
\hline $\begin{array}{l}\text { Slope } \\
\text { Reinforcement }\end{array}$ & X37 & $\begin{array}{l}\text { Landslides / } \\
\text { collapse of side } \\
\text { walls } \\
\end{array}$ & \\
\hline \multirow{3}{*}{ Excavation } & $\mathrm{X} 38$ & $\begin{array}{l}\text { Tools crashing } \\
\text { workers / facilities } \\
\text { around }\end{array}$ & \\
\hline & X39 & $\begin{array}{l}\text { Workers fall into } \\
\text { the excavation }\end{array}$ & \\
\hline & $\mathrm{X} 40$ & $\begin{array}{l}\text { Landslide } \\
\text { excavation }\end{array}$ & \\
\hline \multirow{2}{*}{ Landscapes } & X41 & $\begin{array}{l}\text { Workers hit by } \\
\text { heavy equipment } \\
\text { (backhoe) }\end{array}$ & \\
\hline & $\mathrm{X} 42$ & $\begin{array}{l}\text { The leg is exposed } \\
\text { to the hoe while } \\
\text { doing soil } \\
\text { discharges }\end{array}$ & \\
\hline & $\mathrm{X} 43$ & $\begin{array}{l}\text { Exposed to dump } \\
\text { truck maneuvers }\end{array}$ & \\
\hline \multicolumn{3}{|c|}{ Work Packages: S tockpiling and Compacting } & \\
\hline \multirow[t]{3}{*}{$\begin{array}{l}\text { Stockpiling } \\
\text { and } \\
\text { Compacting } \\
\end{array}$} & X44 & $\begin{array}{l}\text { Workers fell / } \\
\text { clogged due to soil } \\
\text { conditions }\end{array}$ & \\
\hline & $\mathrm{X} 45$ & $\begin{array}{l}\text { Exposed swing } \\
\text { excavator }\end{array}$ & \\
\hline & X46 & $\begin{array}{l}\text { Exposed maneuver } \\
\text { dump truck }\end{array}$ & \\
\hline \multicolumn{3}{|c|}{ Work Package: Anti Termite } & \\
\hline
\end{tabular}




\begin{tabular}{|c|c|c|c|}
\hline \multicolumn{2}{|c|}{$\begin{array}{l}\text { WBS LEVEL } 6 \\
\text { Activities }\end{array}$} & \multicolumn{2}{|c|}{$\begin{array}{l}\text { RIS K VARIABLES } \\
\text { INFLUENCING OSH }\end{array}$} \\
\hline $\begin{array}{l}\text { Procurement } \\
\text { and Installation } \\
\text { of Anti Termite }\end{array}$ & X47 & $\begin{array}{l}\text { Spray ed or doused } \\
\text { with anti-termite } \\
\text { solution }\end{array}$ & \\
\hline \multicolumn{3}{|c|}{$\begin{array}{c}\text { Work Package: Deepth Foundation (Lower } \\
\text { Structure) }\end{array}$} & \\
\hline \multirow{4}{*}{ Erection } & $\mathrm{X} 48$ & $\begin{array}{l}\text { Worker struck by a } \\
\text { stake }\end{array}$ & \\
\hline & X49 & $\begin{array}{l}\text { Worker picked up } \\
\text { sling crane }\end{array}$ & \\
\hline & X50 & $\begin{array}{l}\text { Crushed pile } \\
\text { during lifting }\end{array}$ & \\
\hline & X51 & $\begin{array}{l}\text { The effect of noise } \\
\text { on the surrounding } \\
\text { environment }\end{array}$ & \\
\hline \multirow{4}{*}{$\begin{array}{l}\text { Pile } \\
\text { Linking }\end{array}$} & $\mathrm{X} 52$ & $\begin{array}{l}\text { Fire due to leaking } \\
\text { tube }\end{array}$ & \\
\hline & X53 & $\begin{array}{l}\text { Respiratory } \\
\text { disorders due to } \\
\text { exposure to } \\
\text { welding fumes }\end{array}$ & \\
\hline & X54 & $\begin{array}{l}\text { Workers suffered } \\
\text { burns due to burst } \\
\text { tubes }\end{array}$ & \\
\hline & X55 & $\begin{array}{l}\text { Irritation to the eye } \\
\text { due to smoke } \\
\text { (Plunged into the } \\
\text { hole during pole } \\
\text { connection) }\end{array}$ & \\
\hline \multirow[b]{2}{*}{$\begin{array}{l}\text { Making } \\
\text { Test Well }\end{array}$} & X56 & $\begin{array}{l}\text { Workers fall into } \\
\text { the excavation }\end{array}$ & \\
\hline & X57 & $\begin{array}{l}\text { Tools crashing } \\
\text { workers / facilities } \\
\text { around }\end{array}$ & \\
\hline \multirow{4}{*}{$\begin{array}{l}\text { Drilling (Wash } \\
\text { Drilling / Dry } \\
\text { Drilling) }\end{array}$} & X58 & $\begin{array}{l}\text { Workers fall while } \\
\text { doing lubrication } \\
\text { on the drilling tool }\end{array}$ & \\
\hline & X59 & $\begin{array}{l}\text { Workers fall while } \\
\text { refueling the } \\
\text { drilling machine }\end{array}$ & \\
\hline & X60 & $\begin{array}{l}\text { Drilling tools } \\
\text { crashing into } \\
\text { workers / facilities }\end{array}$ & \\
\hline & X61 & $\begin{array}{l}\text { Road dirty / } \\
\text { slippery due to } \\
\text { spilled soil }\end{array}$ & \\
\hline \multirow{2}{*}{$\begin{array}{l}\text { Installation } \\
\text { of Casing }\end{array}$} & X62 & $\begin{array}{l}\text { Worker struck by } \\
\text { reinforcement }\end{array}$ & \\
\hline & $\mathrm{X} 63$ & $\begin{array}{l}\text { Worker stricken } \\
\text { casing }\end{array}$ & \\
\hline $\begin{array}{l}\text { Drilling uses } \\
\text { mud fluid }\end{array}$ & X64 & $\begin{array}{l}\text { Splashed or } \\
\text { flushed by fluid }\end{array}$ & \\
\hline \multirow[t]{2}{*}{$\begin{array}{l}\text { Cleaning of } \\
\text { drill holes }\end{array}$} & X65 & $\begin{array}{l}\text { Skin dermatitis due } \\
\text { to dust and smoke }\end{array}$ & \\
\hline & X66 & $\begin{array}{l}\text { Eye irritation due } \\
\text { to smoke }\end{array}$ & \\
\hline \multirow{2}{*}{$\begin{array}{l}\text { Installation of } \\
\text { Bored Pile } \\
\text { Repetition }\end{array}$} & X67 & $\begin{array}{l}\text { Workers } \\
\text { puntured iron }\end{array}$ & \\
\hline & X68 & $\begin{array}{l}\text { Worker hands are } \\
\text { exposed to an iron }\end{array}$ & \\
\hline
\end{tabular}

\begin{tabular}{|c|c|c|c|}
\hline \multicolumn{2}{|c|}{$\begin{array}{l}\text { WBS LEVEL } 6 \\
\text { Activities }\end{array}$} & \multicolumn{2}{|c|}{$\begin{array}{c}\text { RIS K VARIABLES } \\
\text { INFLUENCING OSH }\end{array}$} \\
\hline & & $\begin{array}{l}\text { cutting machine } \\
\text { (bar cutter or bar } \\
\text { bender) }\end{array}$ & \\
\hline $\begin{array}{l}\text { Rebuild with a } \\
\text { vacuum }\end{array}$ & X69 & $\begin{array}{l}\text { Skin dermatitis due } \\
\text { to dust and smoke }\end{array}$ & \\
\hline \multirow{2}{*}{$\begin{array}{l}\text { Installation of } \\
\text { tremie pipes for } \\
\text { casting }\end{array}$} & $\mathrm{X} 70$ & $\begin{array}{l}\text { Tools crashing into } \\
\text { nearby workers / } \\
\text { facilities }\end{array}$ & \\
\hline & X71 & $\begin{array}{l}\text { Pipe off / fall into } \\
\text { the boring hole } \\
\text { casting drill }\end{array}$ & \\
\hline \multirow{2}{*}{$\begin{array}{l}\text { Casting drill } \\
\text { pole }\end{array}$} & $\mathrm{X} 72$ & $\begin{array}{l}\text { Traffic congestion } \\
\text { due to the truck } \\
\text { mixer queue on the } \\
\text { drill biting work }\end{array}$ & \\
\hline & X73 & $\begin{array}{l}\text { Tremie pipes } \\
\text { jammed so } \\
\text { concrete setting } \\
\text { with casing }\end{array}$ & \\
\hline $\begin{array}{l}\text { Withdrawal of } \\
\text { the casing }\end{array}$ & X74 & $\begin{array}{l}\text { Worker stricken } \\
\text { casing when } \\
\text { making withdrawal }\end{array}$ & \\
\hline \multicolumn{4}{|c|}{$\begin{array}{l}\text { Work Package: } \text { Test Pile } \\
\end{array}$} \\
\hline $\begin{array}{l}\text { Installation } \\
\text { of cross } \\
\text { beam }\end{array}$ & $\mathrm{X} 75$ & $\begin{array}{l}\text { Injured by } \\
\text { equipment }\end{array}$ & \\
\hline Loading & $\mathrm{X} 76$ & $\begin{array}{l}\text { Overwritten } \\
\text { material }\end{array}$ & \\
\hline $\begin{array}{l}\text { Drilling } \\
\text { pole }\end{array}$ & $\mathrm{X} 77$ & $\begin{array}{l}\text { Accident due to } \\
\text { boring tool } \\
\text { collapsed and } \\
\text { overturned on } \\
\text { drilling work }\end{array}$ & \\
\hline $\begin{array}{l}\text { Installation } \\
\text { of PDA } \\
\text { tools Test }\end{array}$ & $\mathrm{X} 78$ & $\begin{array}{l}\text { Wounded by } \\
\text { equipment }\end{array}$ & \\
\hline \multicolumn{4}{|c|}{ Work Package: Diaphragm Wall } \\
\hline \multirow{3}{*}{ Grab soil } & X79 & $\begin{array}{l}\text { Tools crashing } \\
\text { workers / facilities } \\
\text { around }\end{array}$ & \\
\hline & $\mathrm{X} 80$ & $\begin{array}{l}\text { Workers fall into } \\
\text { the excavation }\end{array}$ & \\
\hline & $\mathrm{X} 81$ & $\begin{array}{l}\text { Landslide } \\
\text { excavation }\end{array}$ & \\
\hline Landscapes & $\mathrm{X} 82$ & $\begin{array}{l}\text { Workers fall into } \\
\text { the pit }\end{array}$ & \\
\hline $\begin{array}{l}\text { Pouring Slurry } \\
\text { Fluid }\end{array}$ & X83 & $\begin{array}{l}\text { Eyes splashed or } \\
\text { watered solution }\end{array}$ & \\
\hline $\begin{array}{l}\text { Precast Panel } \\
\text { Installation }\end{array}$ & $\mathrm{X} 84$ & $\begin{array}{l}\text { Overwritten } \\
\text { material precast at } \\
\text { lifting material }\end{array}$ & \\
\hline \multirow[t]{2}{*}{ Spawning } & $\mathrm{X} 85$ & $\begin{array}{l}\text { Enlargement } \\
\text { Worker hands are } \\
\text { exposed to a cut- } \\
\text { iron machine (bar } \\
\text { cutter or bar } \\
\text { bender) during } \\
\text { fabrication }\end{array}$ & \\
\hline & $\mathrm{X} 86$ & Working legs & \\
\hline
\end{tabular}




\begin{tabular}{|c|c|c|c|}
\hline \multicolumn{2}{|c|}{$\begin{array}{l}\text { WBS LEVEL } 6 \\
\text { Activities }\end{array}$} & \multicolumn{2}{|c|}{$\begin{array}{l}\text { RISK VARIABLES } \\
\text { INFLUENCING OSH }\end{array}$} \\
\hline & & wedged in iron & \\
\hline & & $\begin{array}{l}\text { The hands of the } \\
\text { workers is stabbed }\end{array}$ & \\
\hline & $\mathrm{X} 87$ & with wire & \\
\hline & $\mathrm{X} 88$ & $\begin{array}{l}\text { The hands of } \\
\text { workers exposed } \\
\text { pliers when tying } \\
\text { the ring }\end{array}$ & \\
\hline & X89 & Stumbled iron & \\
\hline \multirow{7}{*}{ Formwork } & \multirow{2}{*}{ X90 } & $\begin{array}{l}\text { Overwritten } \\
\text { Formwork }\end{array}$ & \\
\hline & & \multirow{2}{*}{$\begin{array}{l}\text { Workers are } \\
\text { hammered }\end{array}$} & \\
\hline & X91 & & \\
\hline & X92 & $\begin{array}{l}\text { Workers fall down } \\
\text { due to porous } \\
\text { wood }\end{array}$ & \\
\hline & X93 & $\begin{array}{l}\text { Workers are } \\
\text { stricken with } \\
\text { material }\end{array}$ & \\
\hline & X94 & $\begin{array}{l}\text { Punctured legs of } \\
\text { messy material }\end{array}$ & \\
\hline & X95 & $\begin{array}{l}\text { Legs scratched } \\
\text { cluttered material }\end{array}$ & \\
\hline \multirow{4}{*}{ Concretion } & X96 & $\begin{array}{l}\text { Sprayed or sprayed } \\
\text { with concrete }\end{array}$ & \\
\hline & X97 & $\begin{array}{l}\text { The concrete } \\
\text { bucket fell off the } \\
\text { TC due to the sling } \\
\text { severed so that it } \\
\text { spilled over the } \\
\text { worker underneath }\end{array}$ & \\
\hline & X98 & $\begin{array}{l}\text { Traffic congestion } \\
\text { due to truck mixer } \\
\text { queue at casting } \\
\text { job diapraghma } \\
\text { wall }\end{array}$ & \\
\hline & X99 & $\begin{array}{l}\text { Ready mix from } \\
\text { concrete pump } \\
\text { spilled on workers }\end{array}$ & \\
\hline \multicolumn{3}{|c|}{$\begin{array}{c}\text { Work Packages: Soldier Pile, Contiguous } \\
\text { Pile, Secant Pile }\end{array}$} & \\
\hline $\begin{array}{l}\text { Drilling for } \\
\text { concrete pile on } \\
\text { soldier pile }\end{array}$ & $\mathrm{X} 100$ & $\begin{array}{l}\text { Accident due to } \\
\text { boring tool } \\
\text { collapsed and } \\
\text { overturned on } \\
\text { work Soldier Pile, } \\
\text { Contiguous Pile, } \\
\text { Secant Pile }\end{array}$ & \\
\hline $\begin{array}{l}\text { Drilling } \\
\text { bentonite pile } \\
\text { on contiguous } \\
\text { pile }\end{array}$ & $\mathrm{X} 101$ & $\begin{array}{l}\text { Holes filled with } \\
\text { bentonite fluid left } \\
\text { open not closed } \\
\text { sheeting tarpaulin }\end{array}$ & \\
\hline $\begin{array}{l}\text { Drilling for } \\
\text { boneless } \\
\text { concrete on } \\
\text { secant pile }\end{array}$ & $\mathrm{X} 102$ & $\begin{array}{l}\text { The drilling mud } \\
\text { disposal is not } \\
\text { considered }\end{array}$ & \\
\hline \multicolumn{4}{|c|}{ Work Package: Ground Anchor } \\
\hline Soil drilling & $\mathrm{X} 103$ & $\begin{array}{l}\text { Workers fall while } \\
\text { performing }\end{array}$ & \\
\hline
\end{tabular}

\begin{tabular}{|c|c|c|c|}
\hline \multicolumn{2}{|c|}{$\begin{array}{l}\text { WBS LEVEL } 6 \\
\text { Activities }\end{array}$} & \multicolumn{2}{|c|}{$\begin{array}{c}\text { RIS K VARIABLES } \\
\text { INFLUENCING OSH }\end{array}$} \\
\hline & & $\begin{array}{l}\text { lubrication on the } \\
\text { drilling tool }\end{array}$ & \\
\hline & X104 & $\begin{array}{l}\text { Workers fall while } \\
\text { refueling the } \\
\text { drilling machine }\end{array}$ & \\
\hline Drilling & $\mathrm{X} 105$ & $\begin{array}{l}\text { Accident due to } \\
\text { boring tool } \\
\text { collapsed and } \\
\text { overturned on } \\
\text { ground anchor } \\
\text { work }\end{array}$ & \\
\hline \multicolumn{3}{|c|}{$\begin{array}{c}\text { Work Packages: Raft Foundation, Pile Cap, } \\
\text { Tie Beam, Retaining Wall, Ramp, } \\
\text { GWT/RWT }\end{array}$} & \\
\hline \multirow{6}{*}{ Spawning } & X106 & $\begin{array}{l}\text { The iron } \\
\text { scaffolding is not } \\
\text { solid }\end{array}$ & \\
\hline & X107 & $\begin{array}{l}\text { Iron material falls } \\
\text { when lifting } \\
\text { material with TC }\end{array}$ & \\
\hline & $\mathrm{X} 108$ & $\begin{array}{l}\text { Slip on the way up } \\
\text { the column }\end{array}$ & \\
\hline & $\mathrm{X} 109$ & $\begin{array}{l}\text { Worker hands } \\
\text { stabbed wire }\end{array}$ & \\
\hline & $\mathrm{X} 110$ & $\begin{array}{l}\text { Workers hands hit } \\
\text { by pliers while } \\
\text { tying the ring }\end{array}$ & \\
\hline & X111 & Triple iron & \\
\hline \multirow{6}{*}{ Formwork } & $\mathrm{X} 112$ & $\begin{array}{l}\text { Overwritten } \\
\text { Formwork }\end{array}$ & \\
\hline & $\mathrm{X} 113$ & $\begin{array}{l}\text { Workers are } \\
\text { hammered }\end{array}$ & \\
\hline & $\mathrm{X} 114$ & $\begin{array}{l}\text { Workers fall down } \\
\text { due to porous } \\
\text { wood }\end{array}$ & \\
\hline & $\mathrm{X} 115$ & $\begin{array}{l}\text { Workers are } \\
\text { stricken with } \\
\text { material }\end{array}$ & \\
\hline & X116 & $\begin{array}{l}\text { Legs exposed nails } \\
\text { dismantling } \\
\text { formwork form } \\
\text { work }\end{array}$ & \\
\hline & $\mathrm{X} 117$ & $\begin{array}{l}\text { Legs scratched } \\
\text { cluttered material }\end{array}$ & \\
\hline \multirow{4}{*}{ Concretion } & X118 & $\begin{array}{l}\text { Pipe concrete } \\
\text { pump clogged then } \\
\text { broke and exposed } \\
\text { workers }\end{array}$ & \\
\hline & X119 & $\begin{array}{l}\text { Overloaded } \\
\text { concrete bucket } \\
\text { drops from TC due } \\
\text { to slashed sling so } \\
\text { it spills over the } \\
\text { worker underneath }\end{array}$ & \\
\hline & $\mathrm{X} 120$ & Traffic congestion & \\
\hline & $\mathrm{X} 121$ & $\begin{array}{l}\text { Due to truck mixer } \\
\text { queue at foundry }\end{array}$ & \\
\hline
\end{tabular}




\begin{tabular}{|c|c|c|c|}
\hline \multicolumn{2}{|c|}{$\begin{array}{l}\text { WBS LEVEL } 6 \\
\text { Activities }\end{array}$} & \multicolumn{2}{|c|}{$\begin{array}{c}\text { RIS K VARIABLES } \\
\text { INFLUENCING OSH }\end{array}$} \\
\hline & & $\begin{array}{l}\text { work raft } \\
\text { foundation, pile } \\
\text { cap, tie beam, } \\
\text { retaining wall, } \\
\text { ramp, gwt / rwt }\end{array}$ & \\
\hline $\begin{array}{l}\text { Procurement \& } \\
\text { Installation of }\end{array}$ & $\mathrm{X} 122$ & $\begin{array}{l}\text { Ready mix from } \\
\text { concrete pump } \\
\text { spilled on workers }\end{array}$ & \\
\hline \multirow[t]{3}{*}{$\begin{array}{l}\text { Precast } \\
\text { Concrete }\end{array}$} & X123 & $\begin{array}{l}\text { Hook / sling TC } \\
\text { disconnected when } \\
\text { transporting } \\
\text { precast raft } \\
\text { foundation, pile } \\
\text { cap, tie beam, } \\
\text { retaining wall, } \\
\text { ramp, gwt / rwt }\end{array}$ & \\
\hline & X124 & $\begin{array}{l}\text { Form wrok } \\
\text { collapse on the } \\
\text { installation of } \\
\text { precast raft } \\
\text { foundation, pile } \\
\text { cap, tie beam, } \\
\text { retaining wall, } \\
\text { ramp, gwt / rwt }\end{array}$ & \\
\hline & $\mathrm{X} 125$ & $\begin{array}{l}\text { Workers fall from } \\
\text { altitude on the } \\
\text { installation of } \\
\text { precast raft } \\
\text { foundations, piles, } \\
\text { tie beam, retaining } \\
\text { walls, ramps, gwt / } \\
\text { rwt }\end{array}$ & \\
\hline \multicolumn{3}{|c|}{$\begin{array}{c}\text { Work Packages: Columns, Beams, Plates, } \\
\text { Ladders, Core wall / S hear wall (Upper } \\
\text { Structure) }\end{array}$} & \\
\hline \multirow{7}{*}{ Spawning } & $\mathrm{X} 126$ & $\begin{array}{l}\text { Worker hands are } \\
\text { exposed to an iron } \\
\text { cutting machine } \\
\text { (bar cutter or bar } \\
\text { bender) when } \\
\text { manufacturing iron } \\
\text { for upper structure }\end{array}$ & \\
\hline & $\mathrm{X} 127$ & $\begin{array}{l}\text { Worker legs } \\
\text { wedged in iron }\end{array}$ & \\
\hline & & $\begin{array}{l}\text { Slip on the way up } \\
\text { the column }\end{array}$ & \\
\hline & $\mathrm{X} 128$ & $\begin{array}{l}\text { Worker hands } \\
\text { stabbed wire }\end{array}$ & \\
\hline & X129 & & \\
\hline & $\mathrm{X} 130$ & $\begin{array}{l}\text { Workers hands hit } \\
\text { by tweezers while } \\
\text { tying the ring }\end{array}$ & \\
\hline & $\mathrm{X} 131$ & Triple iron & \\
\hline \multirow{2}{*}{ Formwork } & $\mathrm{X} 132$ & $\begin{array}{l}\text { Overwritten } \\
\text { Formwork }\end{array}$ & \\
\hline & $\mathrm{X} 133$ & $\begin{array}{l}\text { Workers are } \\
\text { hammered }\end{array}$ & \\
\hline
\end{tabular}

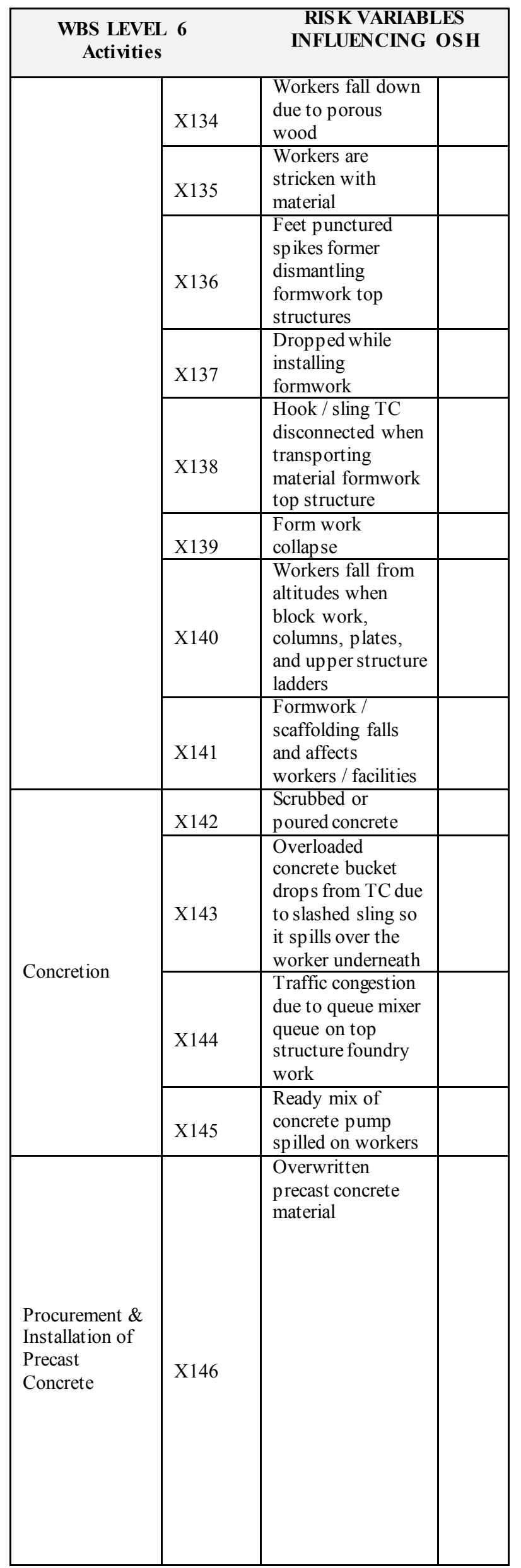




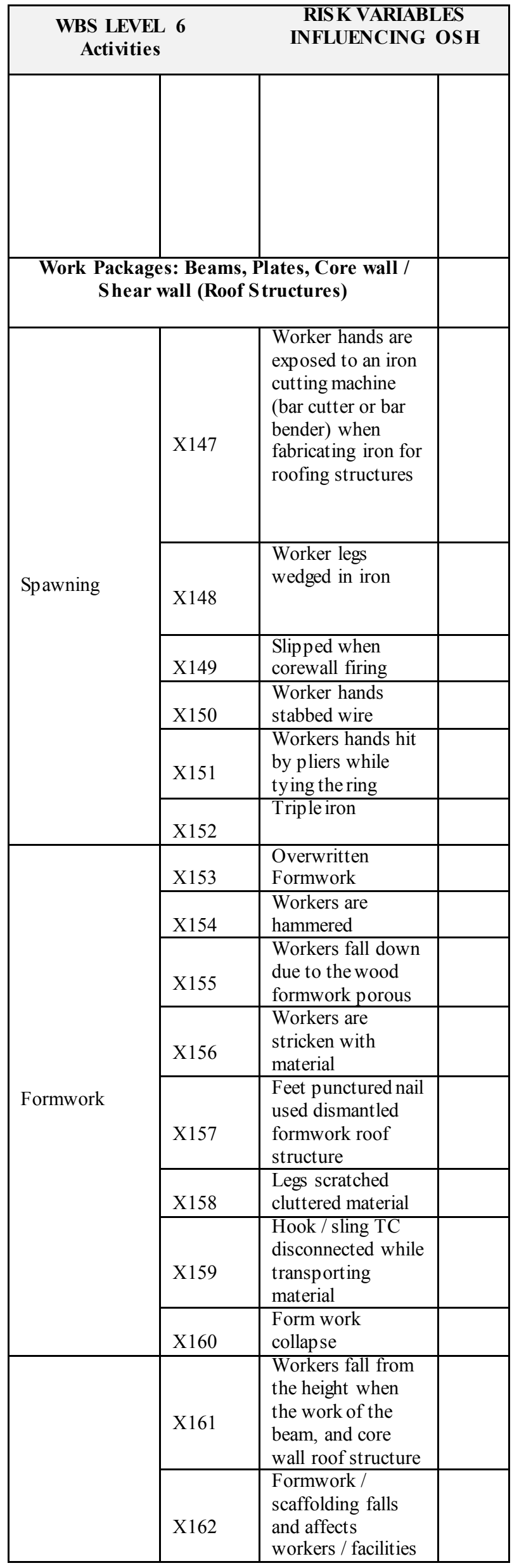

\begin{tabular}{|c|c|c|c|}
\hline \multicolumn{2}{|c|}{$\begin{array}{c}\text { WBS LEVEL } 6 \\
\text { Activities }\end{array}$} & \multicolumn{2}{|c|}{$\begin{array}{l}\text { RISK VARIABLES } \\
\text { INFLUENCING OSH }\end{array}$} \\
\hline Concretion & $\mathrm{X} 163$ & $\begin{array}{l}\text { Sprayed or sprayed } \\
\text { with concrete }\end{array}$ & \\
\hline & X164 & $\begin{array}{l}\text { Overloaded } \\
\text { concrete bucket } \\
\text { drops from TC due } \\
\text { to slashed sling so } \\
\text { it spills over the } \\
\text { worker underneath } \\
\end{array}$ & \\
\hline & $\mathrm{X} 165$ & Traffic congestion & \\
\hline & X166 & $\begin{array}{l}\text { Due to the truck } \\
\text { mixer queue at the } \\
\text { foundry work of } \\
\text { the roof structure }\end{array}$ & \\
\hline \multirow{4}{*}{$\begin{array}{l}\text { Procurement \& } \\
\text { Installation of } \\
\text { Precast } \\
\text { Concrete }\end{array}$} & X167 & $\begin{array}{l}\text { Overwritten } \\
\text { precast concrete } \\
\text { material }\end{array}$ & \\
\hline & X168 & $\begin{array}{l}\text { Hook / sling TC } \\
\text { disconnects when } \\
\text { transporting } \\
\text { precast roofing } \\
\text { material }\end{array}$ & \\
\hline & X169 & $\begin{array}{l}\text { Form work } \\
\text { collapse }\end{array}$ & \\
\hline & $\mathrm{X} 170$ & $\begin{array}{l}\text { Workers fell from } \\
\text { the height during } \\
\text { the installation of } \\
\text { precast roof } \\
\text { structure }\end{array}$ & \\
\hline \multirow{3}{*}{$\begin{array}{l}\text { Installation of } \\
\text { roof covers }\end{array}$} & $\mathrm{X} 171$ & $\begin{array}{l}\text { Workers exp osed } \\
\text { to sunlight while } \\
\text { working on the } \\
\text { roof structure }\end{array}$ & \\
\hline & $\mathrm{X} 172$ & $\begin{array}{l}\text { Workers fall from } \\
\text { height during } \\
\text { erection of roof } \\
\text { steel }\end{array}$ & \\
\hline & $\mathrm{X} 173$ & $\begin{array}{l}\text { Workers fall from } \\
\text { altitude when } \\
\text { helipad plates } \\
\text { work }\end{array}$ & \\
\hline
\end{tabular}

Data collection was carried out using questionnaire survey to understand the perception of the practitioners to the risk factors. Once the probabilities and impacts are determined, the risk score can be calculated with following :

$$
R=P x I
$$

Where $\mathrm{R}=$ risk factor, $\mathrm{P}=$ probability and $\mathrm{I}=$ impact.

The probability and impact matrix or risk level matrix (Table 3 and Table 4) illustrates a risk rating assignment for risk factors.

This analysis is done on the results of questionnaires given to respondents by using Likert measurement scale. The probability value of the occurrence of risk and the impact generated multiplied to produce a number that can be made ranking of risk factors. The risk matrix shows the combination of impact and probability as shown below: 
Table 3. Probability Matrix

\begin{tabular}{|c|c|c|c|c|c|}
\hline Grade & 1 & 2 & 3 & 4 & 5 \\
\hline $\begin{array}{c}\text { Probability } \\
\text { Criteria }\end{array}$ & $\begin{array}{c}\text { Very } \\
\text { Low }\end{array}$ & Low & Moderate & High & $\begin{array}{c}\text { Very } \\
\text { High }\end{array}$ \\
\hline Threats & 0,10 & 0,30 & 0,50 & 0,70 & 0,90 \\
\hline
\end{tabular}

Table 4. Impact Matrix

\begin{tabular}{|c|c|c|c|c|c|}
\hline Grade & 1 & 2 & 3 & 4 & 5 \\
\hline Impact & $\begin{array}{c}\text { No } \\
\text { Criteria } \\
\text { Influ } \\
\text { entia } \\
1\end{array}$ & $\begin{array}{c}\text { Less } \\
\text { Influ } \\
\text { ential }\end{array}$ & $\begin{array}{c}\text { Quite } \\
\text { Influen } \\
\text { tial }\end{array}$ & $\begin{array}{c}\text { Influen } \\
\text { tial }\end{array}$ & $\begin{array}{c}\text { Very } \\
\text { Influen } \\
\text { tial }\end{array}$ \\
\hline Threats & 0,05 & 0,10 & 0,20 & 0,40 & 0,80 \\
\hline
\end{tabular}

\section{$5 \quad$ Result and Discussion}

The results of this study are as follows:

1. To answer RQ 1 found WBS Building Standard Building in Figure 2. WBS Standard For High Rise Building

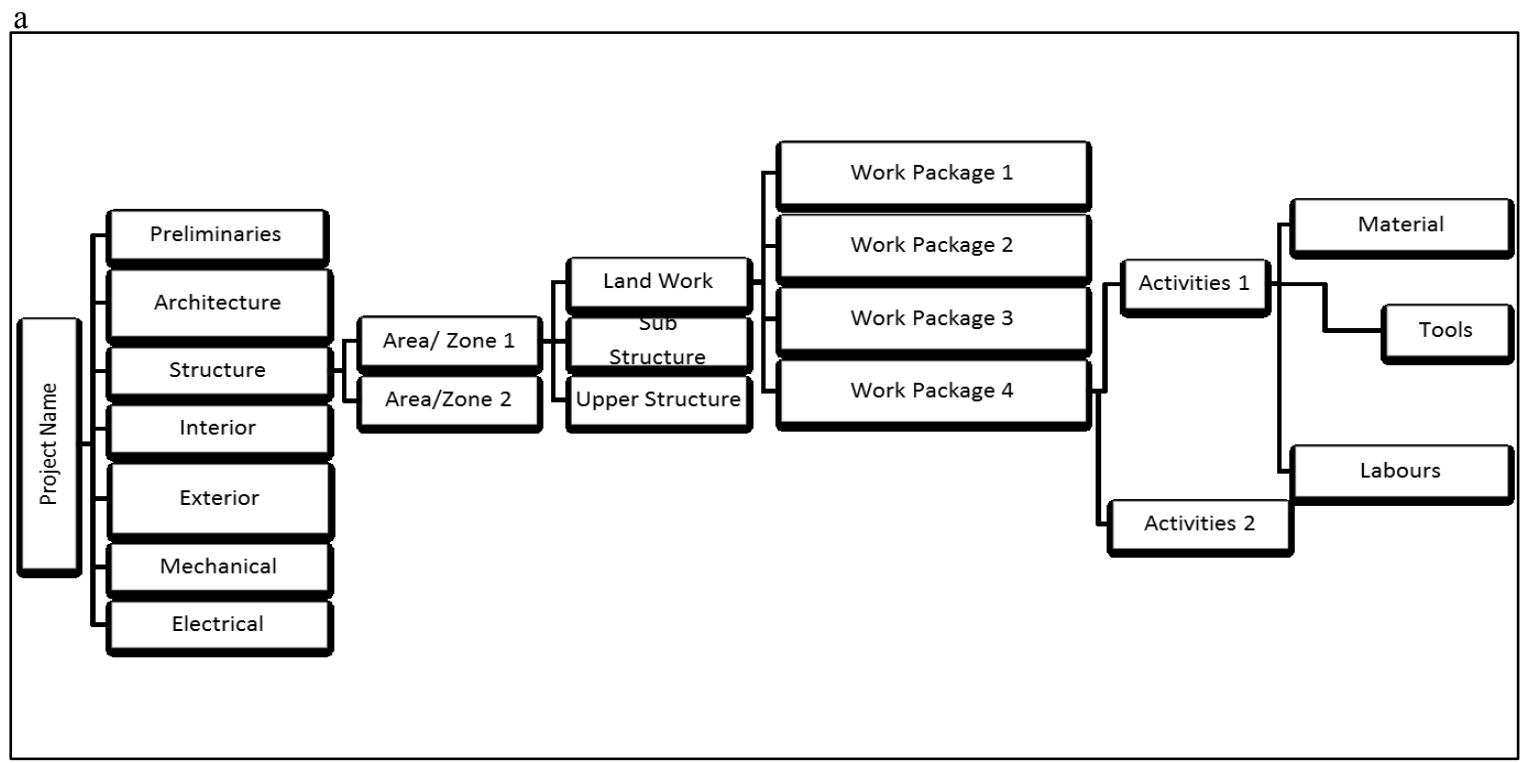

Fig 2. WBS Standard For High Rise Building

Level 1 is for the name of project, level 2 is work sections, preliminaries, structure, architecture, external work, interior, mechanical and electrical works. Level 3 is for area, level 4 are for sub work sections among others soil work, sub structure work, upper structure work, and roof work. Level 5 is for work package, level 6 is for activities, and level 7 is for resources.

2. To answer RQ 2 there is a potentially hazardous source of risk and affect the performance of OSH on Structural Work in Table 5. Dominant Risk as follows:

Table 5. Dominant Risk

\begin{tabular}{|c|c|l|c|c|}
\hline No & \multicolumn{2}{|c|}{ Risk Variables Influencing } & Rank & $\begin{array}{l}\text { Lev } \\
\text { el }\end{array}$ \\
\hline 1 & X9 & $\begin{array}{l}\text { Tool crashing into } \\
\text { nearby worker / } \\
\text { facility (bulldozer } \\
\text { and combine } \\
\text { harvester) at ground } \\
\text { level }\end{array}$ & 23 & H \\
\hline 2 & X15 & $\begin{array}{l}\text { Worker fell into the } \\
\text { excavation }\end{array}$ & 13 & $\mathrm{H}$ \\
\hline
\end{tabular}

\begin{tabular}{|c|c|l|c|c|}
\hline No & \multicolumn{2}{|c|}{ Risk Variables Influencing } & Rank & $\begin{array}{l}\text { Lev } \\
\text { el }\end{array}$ \\
\hline 3 & X16 & $\begin{array}{l}\text { Landslide } \\
\text { excavation }\end{array}$ & H \\
\hline 4 & X27 & $\begin{array}{l}\text { Material regardless } \\
\text { of the hook/ sling } \\
\text { mobile crane is } \\
\text { broken }\end{array}$ & 16 & $\mathrm{H}$ \\
\hline 5 & $\begin{array}{l}\text { Hook / sling TC } \\
\text { disconnected when } \\
\text { transporting precast } \\
\text { raft foundation, pile } \\
\text { cap, tie beam, } \\
\text { retaining wall, }\end{array}$ & 21 & $\mathrm{H}$ \\
\hline
\end{tabular}




\begin{tabular}{|c|c|c|c|c|}
\hline \multirow[t]{2}{*}{ No } & \multicolumn{2}{|c|}{$\begin{array}{c}\text { Risk Variables Influencing } \\
\text { OSH } \\
\end{array}$} & \multirow[t]{2}{*}{ Rank } & \multirow[t]{2}{*}{$\begin{array}{l}\text { Lev } \\
\text { el }\end{array}$} \\
\hline & & ramp, gwt / rwt & & \\
\hline 6 & X124 & $\begin{array}{l}\text { Form work collapse } \\
\text { on the installation } \\
\text { of precast raft } \\
\text { foundation, pile } \\
\text { cap, tie beam, } \\
\text { retaining wall, } \\
\text { ramp, gwt / rwt }\end{array}$ & 15 & $\mathrm{H}$ \\
\hline 7 & $\mathrm{X} 125$ & $\begin{array}{l}\text { Workers fall from } \\
\text { altitude on the } \\
\text { installation of } \\
\text { precast raft } \\
\text { foundations, piles, } \\
\text { tie beam, retaining } \\
\text { walls, ramps, gwt / } \\
\text { rwt }\end{array}$ & 4 & $\mathrm{H}$ \\
\hline 8 & X135 & $\begin{array}{l}\text { Workers are } \\
\text { stricken with } \\
\text { material }\end{array}$ & 24 & $\mathrm{H}$ \\
\hline 9 & X139 & $\begin{array}{l}\text { Form work collapse } \\
\text { in uppers structure } \\
\text { work }\end{array}$ & 18 & $\overline{\mathrm{H}}$ \\
\hline 10 & $\mathrm{X} 140$ & $\begin{array}{l}\text { Workers fall from } \\
\text { altitudes when } \\
\text { block work, } \\
\text { columns, plates, } \\
\text { and upper structure } \\
\text { ladders }\end{array}$ & 1 & $\mathrm{H}$ \\
\hline 11 & X141 & $\begin{array}{l}\text { Formwork / } \\
\text { scaffolding falls } \\
\text { and affects workers } \\
\text { / facilities }\end{array}$ & 11 & $\mathrm{H}$ \\
\hline 12 & X143 & $\begin{array}{l}\text { Overloaded } \\
\text { concrete bucket } \\
\text { drops from TC due } \\
\text { to slashed sling so it } \\
\text { spills over the } \\
\text { worker underneath }\end{array}$ & 19 & $\mathrm{H}$ \\
\hline 13 & X146 & $\begin{array}{l}\text { Overwritten precast } \\
\text { concrete material }\end{array}$ & 17 & $\mathrm{H}$ \\
\hline 14 & X159 & $\begin{array}{l}\text { Hook / sling TC } \\
\text { disconnected while } \\
\text { transporting } \\
\text { material } \\
\end{array}$ & 9 & $\mathrm{H}$ \\
\hline 15 & $\mathrm{X} 160$ & Form work collapse & 14 & $\mathrm{H}$ \\
\hline 16 & X161 & $\begin{array}{l}\text { Workers fall from } \\
\text { the height when the } \\
\text { work of the beam, } \\
\text { and core wall roof } \\
\text { structure }\end{array}$ & 6 & $\mathrm{H}$ \\
\hline 17 & X162 & $\begin{array}{l}\text { Formwork / } \\
\text { scaffolding falls } \\
\text { and affects workers } \\
\text { / facilities }\end{array}$ & 3 & $\mathrm{H}$ \\
\hline 18 & X164 & Overloaded & 12 & $\mathrm{H}$ \\
\hline
\end{tabular}

\begin{tabular}{|c|c|c|c|c|}
\hline \multirow[t]{2}{*}{ No } & \multicolumn{2}{|c|}{$\begin{array}{c}\text { Risk Variables Influencing } \\
\text { OSH }\end{array}$} & \multirow[t]{2}{*}{ Rank } & \multirow[t]{2}{*}{$\begin{array}{l}\text { Lev } \\
\text { el }\end{array}$} \\
\hline & & $\begin{array}{l}\text { concrete bucket } \\
\text { drops from TC due } \\
\text { to slashed sling so it } \\
\text { spills over the } \\
\text { worker underneath }\end{array}$ & & \\
\hline 19 & X167 & $\begin{array}{l}\text { Overwritten precast } \\
\text { concrete material }\end{array}$ & 20 & $\mathrm{H}$ \\
\hline 20 & X168 & $\begin{array}{l}\text { Hook / sling TC } \\
\text { disconnects when } \\
\text { transporting precast } \\
\text { roofing material }\end{array}$ & 10 & $\mathrm{H}$ \\
\hline 21 & X169 & Form work collapse & 22 & $\mathrm{H}$ \\
\hline 22 & $\mathrm{X} 170$ & $\begin{array}{l}\text { Workers fell from } \\
\text { the height during } \\
\text { the installation of } \\
\text { precast roof } \\
\text { structure }\end{array}$ & 8 & $\mathrm{H}$ \\
\hline 23 & $\mathrm{X} 172$ & $\begin{array}{l}\text { Workers fall from } \\
\text { height during } \\
\text { erection of roof } \\
\text { steel }\end{array}$ & 7 & $\mathrm{H}$ \\
\hline 24 & X173 & $\begin{array}{l}\text { Workers fall from } \\
\text { altitude when } \\
\text { helipad plates work }\end{array}$ & 2 & $\mathrm{H}$ \\
\hline
\end{tabular}

In the next discussion, we will answer the formulation of the second problem in this study that is the source of any risks that are potentially dangerous on the work of building structures. Based on 173 risk factors, there are 24 dominant risk factors in every activity step which have an effect on OSH performance in construction project especially high rise building construction project. Where from 24 indicator variables belonging to high level of risk dominant happened at Upper Structure and Roof Structure that can decrease OSH performance in similar project, while 111 indicator of variable of medium risk level, and 38 indicator of variable belong to low category. From some of the above dominant risk events with a high level of risk level if related to the literature that has been obtained the author is as follows:

a. The variable risk events X125, X140, X161, $\mathrm{X} 170, \mathrm{X} 172$, and $\mathrm{X} 173$, are risk factors for accidents falling from a height or mired in a perforated place. The opinion by Expert who thinks that workers are still less aware of the importance of occupationalsafety when working at high altitude and there are also workers who are still violating the rules to wear PPE (Personal Protective Equipment) such as helmets, glasses, safety shoes, full body safety harness.

b. The variable risk events X141 and X162 are risk factors formwork / scaffolding fall and override workers / facilities on the work of the roof structure. The following analysis of possible causes of collapse from the use of scaffolding is the inability to accept the load to obtain maximum 
results in accordance with the designed, then the use of raw materials with good quality becomes absolutely neces sary.

c. The risk event X 135 on the installation of the hazard formwork fell from a height according to Expert, the control measures undertaken were SOPs to work at altitudes, then creating a solid platform for workers, using rubber boots for workers To avoid slipping while climbing the formwork and monitoring the workers working at altitude.

d. In the work involving Tower Crane on X27, X123, X143, X159, X164, and X168 the dangers of material falling according to Expert shall be made of operational SOP tower crane, inspection SOP, use, and maintenance of sling. the riggers provide precise directions to the TC operator to lower the material appropriately.
3. To answer RQ 3 Preparation of Safety Plan using WBS standard is as follows, based on ROSHK in attachment of Ministerial Regulation 05 / PRT / M / 2014, and has been added with WBS level taken sample at some risk with high potential value in Table 6 Work Safety Plan Structure and taken 3 highest risk for example as follow:

Table 6. Safety Plan For Structural Work

\begin{tabular}{|c|c|c|c|c|c|c|c|}
\hline NO & $\begin{array}{l}\text { WBS } \\
\text { Level } 5 \\
\text { (WP) }\end{array}$ & $\begin{array}{c}\text { WBS Level } \\
6 \\
\text { (Activities) } \\
\end{array}$ & $\begin{array}{c}\text { Hazard } \\
\text { Identification }\end{array}$ & Probability & Impact & $\begin{array}{l}\text { Risk } \\
\text { Level }\end{array}$ & S afety Risk Control \\
\hline $\mathrm{X} 140$ & $\begin{array}{l}\text { Upper } \\
\text { Structur } \\
\text { e Works } \\
\text { (Colum } \\
\text { n, beam, } \\
\text { plate, } \\
\text { core } \\
\text { wall, } \\
\text { stairs) }\end{array}$ & $\begin{array}{l}\text { Formwork } \\
\text { on Upper } \\
\text { Structure }\end{array}$ & $\begin{array}{l}\text { Workers fall from } \\
\text { altitude when } \\
\text { block work, } \\
\text { column, plates, } \\
\text { and upper } \\
\text { structure ladders. }\end{array}$ & 0,35 & 0,55 & $\begin{array}{l}0,288 \\
\text { High }\end{array}$ & $\begin{array}{l}\text { Complete PPE for each worker } \\
\text { Installing OSH signs } \\
\text { Make SOP pairs formwork } \\
\text { upper structure } \\
\text { Socializing OSH Program }\end{array}$ \\
\hline X173 & $\begin{array}{l}\text { Roof } \\
\text { Structur } \\
\text { e Works } \\
\text { Beams, } \\
\text { Plates, } \\
\text { Core } \\
\text { wall / } \\
\text { Shear } \\
\text { wall }\end{array}$ & $\begin{array}{c}\text { Roof } \\
\text { Structure }\end{array}$ & $\begin{array}{l}\text { Workers fall from } \\
\text { the heights when } \\
\text { the helipad plates } \\
\text { work }\end{array}$ & 0,297 & 0,605 & $\begin{array}{l}0,264 \\
\text { High }\end{array}$ & $\begin{array}{l}\text { Complete safety equipment for } \\
\text { each worker } \\
\text { Installing OSH signs }\end{array}$ \\
\hline $\mathrm{X} 162$ & $\begin{array}{l}\text { Roof } \\
\text { Structur } \\
\text { e Works } \\
\text { Beams, } \\
\text { Plates, } \\
\text { Core } \\
\text { wall / } \\
\text { Shear } \\
\text { wall }\end{array}$ & $\begin{array}{l}\text { Roof } \\
\text { Structure } \\
\text { Works } \\
\text { Beams, } \\
\text { Plates, Core } \\
\text { wall / Shear } \\
\text { wall }\end{array}$ & $\begin{array}{l}\text { Formwork / } \\
\text { scaffolding falls } \\
\text { and affects } \\
\text { workers / facilities } \\
\text { on roof structure } \\
\text { work }\end{array}$ & 0,314 & 0,25 & $\begin{array}{l}0,25 \\
\text { High }\end{array}$ & $\begin{array}{l}\text { Check the installation of } \\
\text { formwork } \\
\text { Make SOP pairs of roof } \\
\text { structure }\end{array}$ \\
\hline
\end{tabular}

\section{Conclusion}

Based on the results of testing and analysis that has been done then it can be concluded as follows:

a. Creating WBS building standards divided into 7 levels, Level 1: Project Name, Level 2: Work Section, Level 3: Zoning Area / Location, Level 4: Sub Work Section, Level 5: Work Packages, Level
6: Activity, and Level 7: Resources. WBS structural work standards can be established according to the classified level after collecting and analyzing data / archives based on BOQ and RKS projects and have been validated by related experts.

b. Identify potentially hazardous sources of risk on structural work. There are 24 high risk risk event variables (X9, X15, X16, X27, X123, X124, $\mathrm{X} 125, \mathrm{X} 135, \mathrm{X} 139, \mathrm{X} 149, \mathrm{X} 141, \mathrm{X} 161, \mathrm{X} 162$, X164 and X167, X168, X169, X170, X172, X173) 
are identified to be the dominant risk in every stage of the high rise building development process. Having obtained the risk event variable is also equipped with the risk response of each variable so that the potential danger can be prevented / mitigated.

c. Preparation of safety plan by using risk based WBS standard is completed and developed according to document of safety planning / ROSHK Government Regulation PU 05 / PRT / M / 2014 at occupational safety planning section for building construction work which can be used either as assessment material of service provider auction process and also as guide for contractors in the preparation of safety planning.

\section{Acknowledgements}

The authors would like to express gratitude to University of Indonesia for granting a support through PITTA no 862/UN2.R3.1/HKP.05.00/2017 scheme in order to assist the researchers in completing this research.

\section{$7 \quad$ References}

[1] Aishah Momoh, Rajkumar Roy and Essam Shehab. A Work Breakdown Structure for Implementing and Costing ERP Project. (Communications of the IBIMA,2008) 94103

[2] Al-Anbari.S, Khalina, A. Safety and Health Risk. Ass.at.Oman. Build. Const. Pro. Oman. IJRET: International Journal of Research in Engineering and Technology eISSN: 23191163 Volume: 02.571-578 (2013).

[3] Alex, Albert. Emerging Strategies for Construction Safety \& Health Hazard Recognition. Journal of Safety, Health \& Environmental Research 152-160, (2014).

[4] Hans, Robert. Wor.Bre.Stru: A Tool for Soft Pro. Sco. Ver. International Journal of Software Engineering \& Applications (IJSEA), Vol.4, No.4. 19-25, (2013).

[5] Kai Chen Goh1,a, Hui Hwang. Accidents Preventive Practice for High-Rise Construction. (EDP Sciences, MATEC Conference, 2016). 04004-p.1-04004-p.6

[6] Kamar, Mohd- N.S. Lop, N. Mat. Contractor's Awareness on Occupational
Safety and Health(OSH) Management Systems in Construction Industry, (EDP Science Confrences, 2014) 01019-p.1-01019p.6,

[7] Mhetre, K; Konnur, B.A; B, Amarsinh. Risk Man.t in. Con. Ind. International Journal of Engineering Research Volume No.5. 153155, (2016)

[8] PMI, Practice Standard for Work Breakdown Structures Second Edition,Pennsylvania: Project Management Institute, (2006).

[9] Satish Kumar. Current State of Construction Safety Planning Practice. Proc. of Int. Conf. on (Advances in Civil Engineering, 2011), 47-50

[10] Shlomo Globerson. Imp. Of. Var. WBS on Pro. Concept. (International Journal of Project Management, 1994), Vol. 12.165-171

[11] Su,Lei. WBS-based Risk Identification for the Whole Process of Real Estate Project and Countermeasure. (National Conference on Information Technology and Computer Science, 2012).780-783

[12] Van Tonder, J.C. and Bekker, M.C. (2001). Analysis of A Methodology to Obtain a Work Breakdown Structure Built Up From Interdependent Key Project Deliverable Packages. (African Rhythm Project Management Conference, 2002),ISBN Number: 0-620-28853-1

[13] Zid Chaher, Ali Raza Soomro. Fac. Risk.Man in Cons. Pro by using Hie. RBS. (International Journal of Scientific and Research Publications, 2016).Volume 6, Issue 7. 703709 